\title{
Soft tissue modelling with conical springs
}

\author{
Nadzeri Omar, ${ }^{\mathrm{a},}$, Yongmin Zhong ${ }^{\mathrm{a}}$, Reza N. Jazar ${ }^{\mathrm{a}}$, Aleksandar Subic ${ }^{\mathrm{b}}$, Julian Smith ${ }^{\mathrm{c}}$ and \\ Bijan Shirinzadeh $^{\mathrm{d}}$ \\ ${ }^{a}$ School of Aerospace, Mechanical and Manufacturing Engineering, RMIT University, PO Box 71, \\ Bundoora, VIC 3083, Australia \\ ${ }^{b}$ Swinburne Research \& Development, Swinburne University of Technology, Hawthorn, VIC 3122, \\ Victoria, Australia \\ ${ }^{c}$ Monash Medical Centre, Monash University, Clayton, VIC 3168, Australia \\ ${ }^{d}$ Department of Mechanical and Aerospace Engineering, Monash University, Clayton, VIC 3800, \\ Australia
}

\begin{abstract}
This paper presents a new method for real-time modelling soft tissue deformation. It improves the traditional massspring model with conical springs to deal with nonlinear mechanical behaviours of soft tissues. A conical spring model is developed to predict soft tissue deformation with reference to deformation patterns. The model parameters are formulated according to tissue deformation patterns and the nonlinear behaviours of soft tissues are modelled with the stiffness variation of conical spring. Experimental results show that the proposed method can describe different tissue deformation patterns using one single equation and also exhibit the typical mechanical behaviours of soft tissues.
\end{abstract}

Keywords: Soft tissue modelling, tissue deformation patterns, stiffness variation, conical spring

\section{Introduction}

Soft tissue deformation is of great importance for surgical simulation. Significant research efforts have been dedicated to modelling of soft tissue deformation. However, modelling of deformable tissues for surgical simulation is difficult because of the conflicting demands of real-time performance and physical accuracy. The finite element method (FEM) is a typical deformation method focusing on physical accuracy. This method uses continuum mechanics to govern the elastic behaviours of soft tissues. In spite of the accuracy, it suffers from being an expensive computational load. Therefore, most of the existing FEMs are dominated by linear elasticity, because linear elastic models allow reduced runtime computations. Although there are some methods based on nonlinear elasticity, these methods can only handle a relatively small number of grid meshes. Additional techniques such as precomputation, matrix condensation, adaptive meshing and GPU acceleration are required to facilitate the computational performance [1-3].

The mass-spring model (MSM) is a typical deformation method focusing on computational

\footnotetext{
* Address for correspondence: Nadzeri Omar, School of Aerospace, Mechanical and Manufacturing Engineering, RMIT University, PO Box 71, Bundoora, VIC 3083, Australia. Tel.: +613 9925 6018; Fax: +613 9925 6108; E-mail: omar.mohdnadzeri@rmit.edu.au.
} 
performance. This method discretises a model based on Hooke's law into discrete mass points connected to each other with massless springs. It is easier to implement in algorithm and is less time consuming in computation. However, it suffers from low accuracy for modelling of soft tissue deformation, as the linear Hooke's law is only suitable for small deformation [4-6], while the behaviours of soft tissues such as human organs are extremely nonlinear [7-9].

Various techniques have been reported focusing on improvement of the MSM's accuracy by introducing nonlinearity. Duffing's equation was used in the MSM to define the nonlinear loaddisplacement response [10-12]. This method divides the deformation curve into two regions. One is linear and the other nonlinear, governed by a linear and nonlinear sub-equation, respectively. However, its performance relies on an optimization process to find the appropriate stiffness constant value. The method also requires the switch between the two sub-equations, leading to an additional computational load.

Combination of springs in a cubical configuration is another approach [13]. The spring orientation is divided into three types; vertices, diagonal and internal diagonal. Each type of spring is allowed to share a common stiffness constant and the selection of stiffness constants depends on the deformation patterns. However, in the case of too many springs, the model becomes stiffer and thus will deform in an unrealistic manner. Xu et al. reported a hybrid method by combining the advantages of MSM and FEM for soft tissue deformation. However, this method requires a large computational load to solve the constitutive equation [14]. Ahmadian and Nikooyan studied an approach using empirical formulae derived from practical experiments for soft tissue deformation, where different soft tissues are assigned to different formulae via an optimization process [15]. However, the accuracy of this method relies on the experimental results on soft tissues. The requirement of experimental results also makes the method difficult to be extensively used. The penetration depth is a method to limit the deformation range of the traditional mass-spring model according to the depth that a surgical tool penetrates in soft tissues [16-18]. In spite of the improved computational performance, there is no physical nonlinearity involved in the method.

This paper presents a new method to predict the nonlinear deformation of soft tissues. This method improves the traditional MSM by replacing the linear spring with the conical spring. It models the nonlinear behaviours of soft tissues using the stiffness variation of conical spring. It also formulates the model parameters according to deformation patterns of soft tissues to improve the simulation realism. Experiments and comparison analysis have been conducted to evaluate the performance of the proposed method.

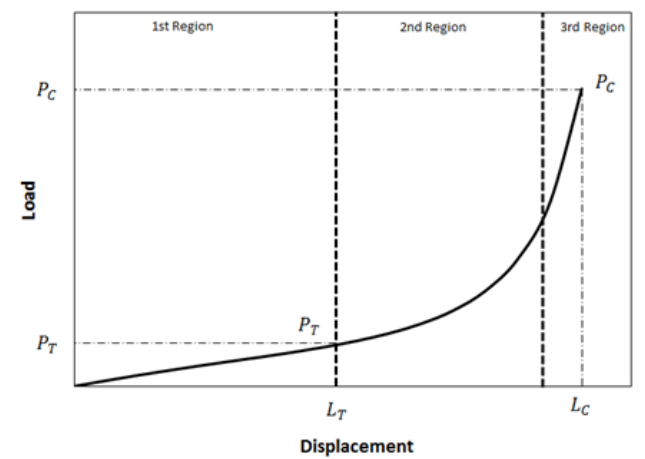

(a)

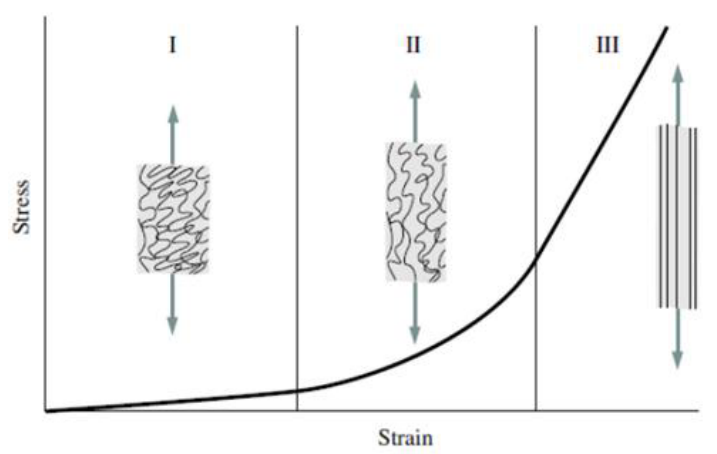

(b)

Fig. 1. The load-displacement response of the conical spring (a) and the stress-strain response of soft tissues (b) [19]. 


\section{Soft tissue modelling}

\subsection{Conical spring derivation}

Typical deformation patterns of soft tissues can be observed in three regions [4, 19]. As shown in Figure 1(b), the first region, which represents small deformation, is linear; the second region is nonlinear, where a greater load is required for soft tissues to deform; then the behaviour is back to a linear pattern in the final region. In order to simulate the nonlinearity and deformation patterns of soft tissues, this paper proposes the use of conical springs instead of linear springs for the MSM.

The conical spring belongs to the helical spring family. It has different sizes of coils, small at the top and getting bigger towards the bottom. Unlike a normal helical spring, the conical spring does not have a single stiffness constant. Its stiffness constant is variable according to the size of the coil. Therefore, the conical spring provides a nonlinear factor for modelling nonlinear behaviours of soft tissues.

There are two types of conical spring: the telescoping and non-telescoping. They have different structures under a fully compressed condition. The former produces a diameter thickness height only while the latter produces a stack-like structure. The former also possesses a greater nonlinearity than the latter. The telescoping and non-telescoping conical springs can be distinguished by the following simple conditions [20]:

$$
\begin{gathered}
\left(D_{2}-D_{1}\right) / 2<n d \quad \begin{array}{c}
\text { for the telescoping conical spring } \\
\left(D_{2}-D_{1}\right) / 2 \geq n d \quad \text { for the non-telescoping conical spring }
\end{array}
\end{gathered}
$$

where $D_{2}$ and $D_{1}$ are the diameters of the largest and the smallest coils, $d$ is the diameter of the wire, and $n$ is the number of coils with the minimum value of 2 .

A simple formulation was proposed by Rodriguez to define the load-displacement relationship of the spring [20]. The formulation is derived using geometrical properties of the spring as shown in Figure 2. This single formulation can be used to describe both the telescoping and non-telescoping spring. The conical spring force $F(u)$ in term of displacement is described as

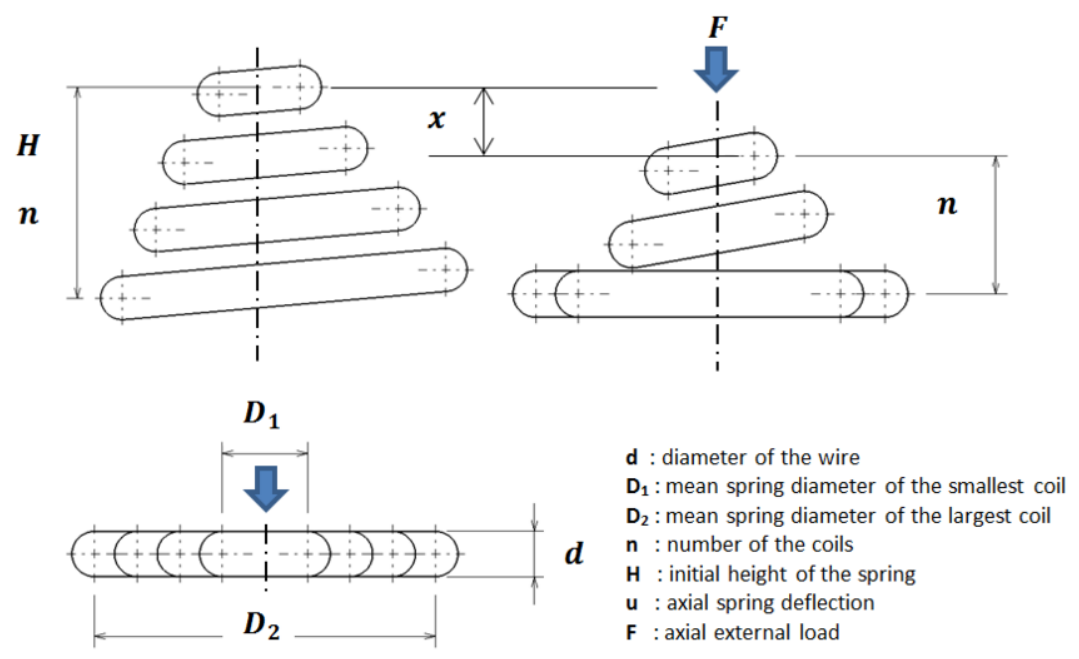

Fig. 2. Parameters use to define conical spring. 


$$
F(u)=\left(K_{1} / 2\right)^{3 / 2}\left[1-\left(1-2\left[1-\left(1+K_{2} / K_{1}^{2}\right)^{1 / 2}\right]\right)^{1 / 2}\right]^{3}
$$

where $K_{1}$ and $K_{2}$ are defined using terms $K_{3}$ to $K_{7}$ as shown in Eqs. (4) to (10).

$$
\begin{gathered}
K_{1}=K_{3}+K_{2} / 3 K_{3} \\
K_{2}=-K_{6} / K_{5} \\
K_{3}=\left[K_{4} / 16+\left[\left(K_{4} / 16\right)^{2}+\left(K_{2} / 3\right)^{3}\right]^{1 / 2}\right]^{1 / 3} \\
K_{4}=\left[\left(K_{7}-u\right) / K_{5}\right]^{2} \\
K_{5}=-\left(2 D_{1}^{4} n\right) /\left[G d^{4}\left(D_{2}-D_{1}\right)\right] \\
K_{6}=-3\left[G d^{4}(H)^{4} / n\right]^{1 / 3} /\left[8\left(D_{2}-D_{1}\right)\right] \\
K_{7}=\left[D_{2}(H)\right] /\left(D_{2}-D_{1}\right)
\end{gathered}
$$

where $H$ is the initial length of the spring, $G$ the torsion modulus and $u$ the displacement of the spring.

Based on the response and the structure of the conical spring, the load-displacement behaviour of the conical spring can be described in three regions. The first region is linear, followed by a nonlinear pattern for the second region and subsequently a linear pattern for the final region. This behaviour exactly matches the deformation patterns of soft tissues. The conical spring deformation is shown in Figure 1(a).

In the first region, the largest coil, which is the most flexible coil, will deform at first. It will deform in the similar condition as a normal helical spring with a single stiffness constant, thus responsible for the first linear region. The next largest coil will deform as soon as the largest coil is fully compressed. At this stage, the largest coil is termed non-active and the second largest coil is the active coil. The process will continue until the smallest coil is active. The gradual change in the diameter of the active coil explains the reason for the variation in stiffness rate and, as a result, a nonlinear response is recorded. Finally, at the smallest coil, since it is the only coil left and the deviation of the previous coils is small, deformation in this region is linear.

Looking on Figure 1(a), the parameters at the transition point (the point that separates the first linear region and the nonlinear region) and the maximum point (the final point on the curve or the maximum load) can be calculated using Eqs. (11) and (12) as:

$$
\begin{aligned}
& F_{C}=\left[G d^{4}(H) /\left(8 D_{1}^{3} n_{a}\right)\right] \\
& F_{T}=\left[G d^{4}(H) /\left(8 D_{2}^{3} n_{a}\right)\right]
\end{aligned}
$$

where $F_{C}$ and $F_{T}$ are the magnitudes of the loads at the at maximum and transition points. The parameters can be described based on the information at these two points.

\subsection{Conical spring based MSM}

The typical dynamic motion of the MSM model is described by Newton's law. The total force experienced by a model consists of an internal force and external force. The internal force is constituted by a damping force and spring elastic force. The damping force is responsible for generating the resistance due to the mass velocity, while the spring elastic force defines the resistance experienced by the spring. When an external force is applied, the dynamic behaviour of each influenced mass point is expressed as 


$$
m_{i} a_{i}+F_{d i}+F_{k i}=F_{\text {ext } \_i}
$$

where $m_{i}$ is the mass at point $i, a_{i}$ the acceleration at point $i, F_{d i}$ the damping force at point $i, F_{k i}$ the spring force at point $i$, and $F_{\text {ext } i}$ the external force at point $i$.

In terms of displacement, the above equation can be rewritten as

$$
m_{i} \ddot{u}_{\imath}+d_{i j} \dot{u}_{i}+K_{i} u_{i}=F_{\text {ext } \_} i
$$

where $m_{i}$ is the mass at point $i$ and $d_{i j}$ is the damping constant between points $i$ and $j$. The terms $u_{i}$, $\dot{u}_{i}$ and $\ddot{u}_{\imath}$ stand for the displacement, velocity and acceleration of point $i$. The spring elastic force at point $i$ is represented as the linear product between the stiffness constant $K_{i}$ and displacement $u_{i}$. The dynamic equation can be solved using the explicit integration method [5-9].

Substituting the linear spring elastic force in Eq. (14) with the conical spring force described by Eq. (3), the proposed conical spring model can be written as

$$
m_{i} \ddot{u}_{\iota}+d_{i j} \dot{u}_{i}+F\left(u_{i}\right)=F_{\text {ext } \_} i
$$

where $F\left(u_{i}\right)$ is the conical spring force.

The proposed method adopts a data-driven strategy to determine the parameters used in the conical spring model from the deformation patterns of soft tissues. In order to define the suitable parameters for the conical spring, parameters are divided into two types: design parameters and operation parameters. Design parameters involve the diameters of the largest and the smallest coils, the number of active coils, the diameter of the wire, the initial height of the spring and the torsion modulus (refer Figure 2). The operation parameters are the mechanical properties and information available at the transition and maximum points from real tissues (refer Figure 1(a)).

The wire diameter $d$ for the normal helical spring can be calculated as [21]

$$
d=D / c
$$

where $c$ is the spring index and $D$ is the mean diameter. The spring index determines the spring strength. The smaller the index is, the stiffer the spring will be. As the minimum value of the spring index is 3 [22], the mean diameter is at least three times greater than $d$.

By considering the diameter of the smallest coil, $D_{1}$, as the mean diameter, the wire diameter for the conical spring can be represented as

$$
d=D_{1} / c
$$

Assuming the initial height $H$ is equal to $L_{C}$ (see Figure 1(a)), the parameters $D_{2}$ and $G$ can be derived from Eqs. (11) and (12).

The different types of conical spring have different minimum and maximum lengths. The minimum length of the telescoping spring, $L_{m i n}$ tel , is equal to the wire diameter $d$

$$
L_{\text {min_tel }}=d
$$

The minimum length of the non-telescoping spring, $L_{\min \_n o n-t e l}$, is the product of the number of 
coils $n$ and the wire diameter $d$

$$
L_{m i n \_n o n-t e l}=n d
$$

The maximum lengths of both telescoping and non-telescoping springs are calculated as follows

$$
\begin{gathered}
L_{\text {max_tel }}=H+(H-d) \\
L_{\text {max_non-tel }}=H+(H-n d)
\end{gathered}
$$

\section{Implementation and discussion}

Experiments have been conducted to evaluate the performance of the proposed method. The performance evaluation focuses on verifying the mechanical properties of soft tissues in terms of the soft tissue deformation patterns [15] and the typical mechanical behaviours of soft tissues [4].

In order to evaluate the realism of the proposed method, a uniaxial loading test was carried out a simple cubical model. The model parameters were designed according to the two types of soft tissue deformation patterns [15]. Figure 3 shows the structure of the cubical model and deformation results.

Figure 4 compares the simulated deformations with the different deformation patterns presented in [15]. It can be seen that the achieved deformation results are in agreement with both deformation
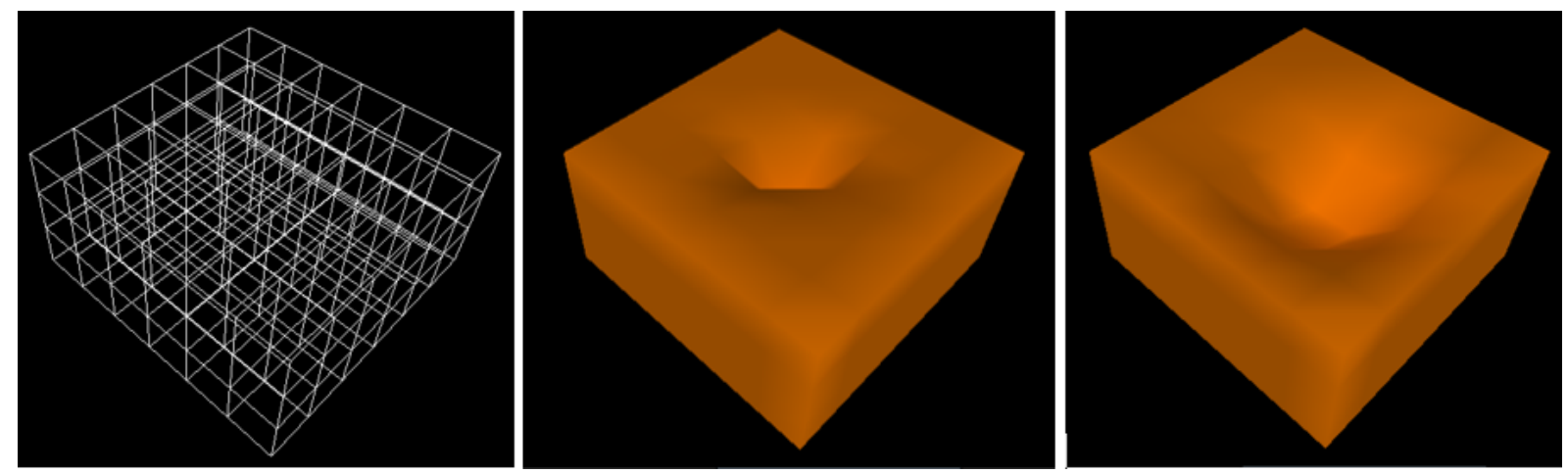

Fig. 3. The topological structure and behaviour of simple cubical model.
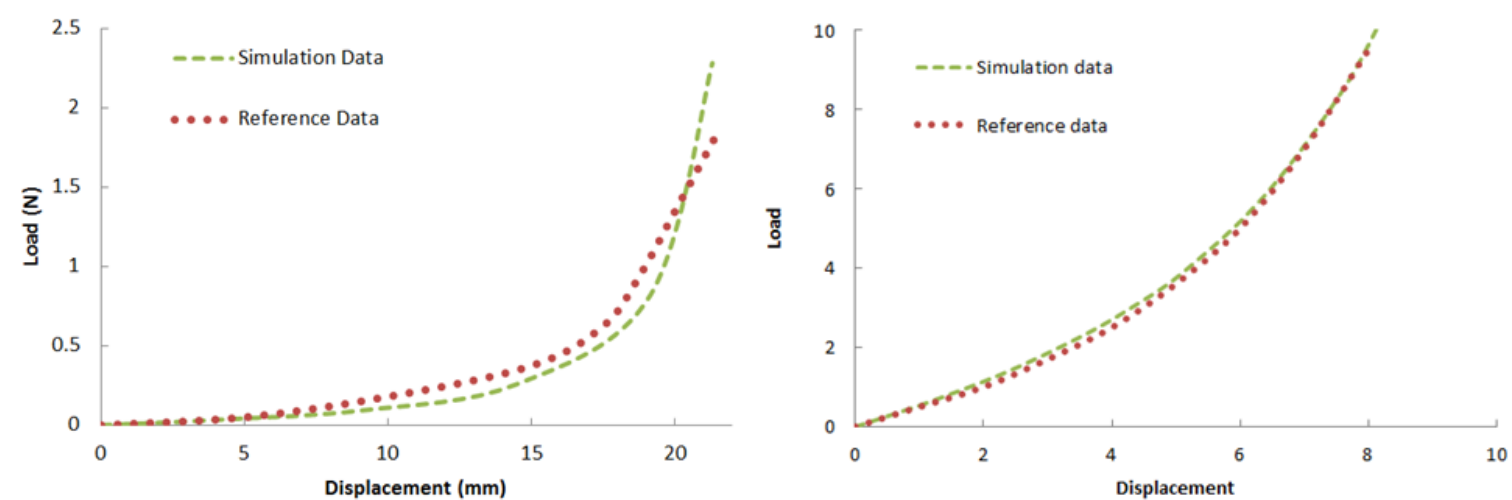

Fig. 4. Comparison of simulated deformations with the different deformation patterns described in [15]. 


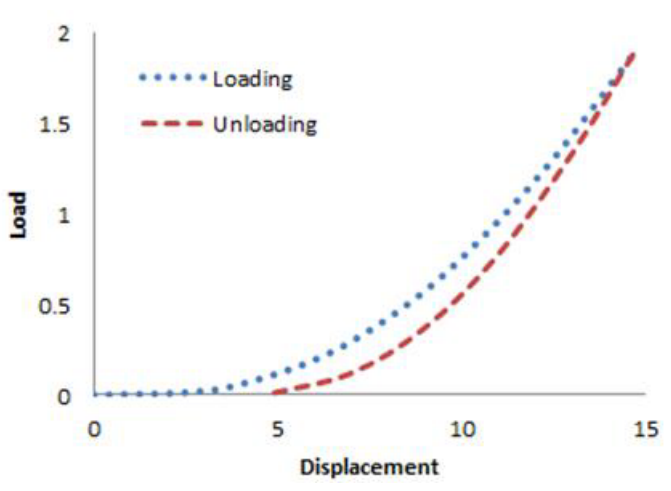

(a)

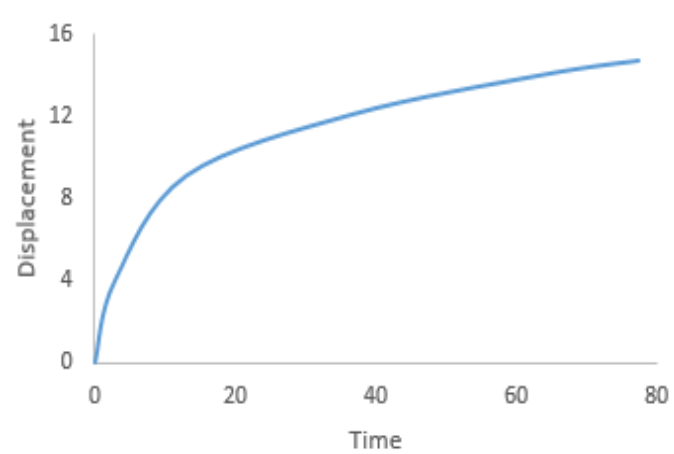

(b)

Fig. 5. The image of the hysteresis loop (a) and the creeping property (b) for the proposed model.

patterns. It demonstrates the proposed method can describe different tissue deformation patterns with one single equation. It also demonstrates the proposed method can achieve the nonlinear loaddeformation relationship.

The typical mechanical behaviours of soft tissues include hysteresis, creeping and nonlinear loaddeformation relationship [4]. In addition to the nonlinear load-deformation relationship demonstrated in Figure 4, trials were also conducted to verify the hysteresis and creeping properties of soft tissues. As shown in Figure 5(a), the loading and unloading processes follow two distinct paths. The area formed by the loop is the energy lost in stretching and releasing soft tissues. It is also observed that the tested subject under the unloading process does not go back to its original position. These are in agreement with the hysteresis property of soft tissues [4]. On the other hand, creeping is the tendency of a material to deform permanently under the influence of continuous stresses. In order to test this property, a constant uniaxial load is applied on the model. Figure 5(b) shows the response of the model to the creep test. The response shows that the model can exhibit the creep property.

\section{Conclusion and future works}

This paper presents a new mass-spring based method to mimic the deformation of soft tissue with reference to deformation patterns of soft tissues. This method introduces conical spring in the traditional MSM model to predict nonlinear mechanical behaviours. It formulates the model parameters according to the deformation patterns of real tissues. The main contributions of the paper are (i) the nonlinear deformation of soft tissues is modelled with the stiffness variation of conical spring rather than a single stiffness constant in the traditional MSM; and (ii) different types of deformation patterns of soft tissues are simulated using just a single equation, leading to the simplicity and improved efficiency for modelling of soft tissue deformation. Experimental results demonstrate that the proposed method is able to describe different deformation patterns of real tissues and also exhibit the typical mechanical properties of soft tissues.

Future research will focus on the improvement of the proposed method by considering the mechanism of load propagation for soft tissue deformation. It is also expected to integrate the proposed method with a haptic device to provide real-time force feedback for surgery simulation. 


\section{References}

[1] H. Courtecuisse, H. Jung, J. Allard, C. Duriez, D.Y. Lee and S. Cotin, GPU-based real-time soft tissue deformation with cutting and haptic feedback, Progress in Biophysics and Molecular Biology 103 (2010), 159-168.

[2] Joldes Grand Roman, Adam Wittek and Karol Miller, Real-time nonlinear finite element computations on GPUApplication to neurosurgical simulation, Computer Methods in Applied Mechanics and Engineering 199 (2010), 33053314.

[3] Z.A. Taylor, M. Cheng and S. Ourselin, High-speed nonlinear finite element analysis for surgical simulation using graphics processing units, IEEE Transactions on Medical Imaging 27 (2008), 650-663.

[4] Y.C. Fung, Biomechanics: Mechanical Properties of Living Tissues, Springer, New York, 1981, pp. 196-257.

[5] Y. Zhong, B. Shirinzadeh, J. Smith and C. Gu, Soft tissue deformation with reaction-diffusion process for surgery simulation, Journal of Visual Languages and Computing 23 (2012), 1-12.

[6] Y. Zhong, B. Shirinzadeh, J. Smith and C. Gu, Thermal-mechanical based soft tissue deformation for surgery simulation, International Journal of Advanced Robotics 24 (2010), 1719-1739.

[7] Y. Zhong, B. Shirinzadeh, J. Smith and C. Gu, An electromechanical based deformable model for soft tissue simulation, Artificial Intelligence in Medicine 47 (2009), 275-288.

[8] Y. Zhong, B. Shirinzadeh, G. Alici and J. Smith, A neural network methodology for deformable object simulation, IEEE Transactions on Information Technology in Biomedicine 10 (2006), 749-762.

[9] Y. Zhong, B. Shirinzadeh, G. Alici and J. Smith, Simulation of deformable models with Poisson equation, Computer Methods in Biomechanics and Biomedical Engineering 9 (2006), 289-304.

[10] T. Cui, A. Song and J. Wu, Simulation of a mass-spring model for global deformation, Frontiers of Electrical and Electronic Engineering in China 4 (2008), 78-82.

[11] F. Chen, L. Gu, P. Huang, J. Zhang and J. Xu, Soft tissue modeling using nonlinear mass spring and simplified medial representation, 29th Annual International Conference of the IEEE Engineering in Medicine and Biology Society 2007 (2007), 5083-5086.

[12] E. Basafa and F. Farahmand, Real-time simulation of the nonlinear visco-elastic deformations of soft tissues, International Journal of Computer Assisted Radiology and Surgery 6 (2011), 297-307.

[13] G. San-Vicente, I. Aguinaga and J. Tomás Celigüeta, Cubical Mass-Spring Model design based on a tensile deformation test and nonlinear material model, IEEE Transactions on Visualization and Computer Graphics 18 (2012), 228-241.

[14] X.P. Liu, S. Xu, H. Zhang and L. Hu, A new hybrid soft tissue model for visio-haptic simulation, IEEE Transactions on Instrumentation and Measurement 60 (2011), 3570-3581.

[15] M.T. Ahmadian and A.A. Nikooyan, Modeling and prediction of soft tissue directional stiffness using in-vitro force, International Journal of Scientific Research 16 (2006), 385-389.

[16] Y.-H. Chang, Y.-T. Chen, C.-W. Chang and C.-L. Lin, Development scheme of haptic-based system for interactive deformable simulation, Computer-Aided Design 42 (2010), 414-424.

[17] K. Choi, H. Sun and P. Heng, An efficient and scalable deformable model for virtual reality-based medical applications, Artificial Intelligence in Medicine 28 (2004), 559-568.

[18] P.H.C. Mun, Y. Zhong, B. Shirinzadeh, J. Smith and C. Gu, An improved mass-spring model for soft tissue deformation with haptic feedback, The 15th Conference on Mechatronics Technology, Melbourne, Australia, 2011, pp. $1-6$.

[19] G.A. Holzapfel, Biomechanics of soft tissue, The Handbook of Materials Behavior Models 3 (2001), 1049-1063.

[20] E. Rodriguez, M. Paredes and M. Sartor, Analytical behavior law for a constant pitch conical compression spring, Journal of Mechanical Design 128 (2006), 1352.

[21] M. Paredes, M. Sartor and C. Masclet, Obtaining an optimal compression spring design directly from a user specification, Proceedings of the Institution of Mechanical Engineers, Part B: Journal of Engineering Manufacture 216 (2002), 419-428.

[22] L.J.A. den Boer, Nonlinear dynamic behaviour of a conical spring with top mass, Master Dissertation, Eindhoven University of Technology, Eindhoven, Netherlands, 2009. 